OPEN ACCESS

Edited by:

Mathieu Letellier,

UMR5297 Institut Interdisciplinaire de Neurosciences (IINS), France

Reviewed by:

Robert J. Kittel,

Leipzig University, Germany

C. Andrew Frank,

University of lowa, United States

${ }^{*}$ Correspondence:

Dion Dickman

dickman@usc.edu

TORCID:

Dion Dickman

orchid.org/0000-0003-1884-284X

Specialty section:

This article was submitted to Cellular Neurophysiology, a section of the journal Frontiers in Cellular

Neuroscience

Received: 15 April 2020 Accepted: 05 June 2020 Published: 26 June 2020

Citation:

Goel P, Nishimura S, Chetlapalli K, Li X, Chen C and Dickman D (2020) Distinct Target-Specific Mechanisms

Homeostatically Stabilize

Transmission at Pre- and

Post-synaptic Compartments.

Front. Cell. Neurosci. 14:196.

doi: 10.3389/fncel.2020.00196

\section{Distinct Target-Specific Mechanisms Homeostatically Stabilize Transmission at Pre- and Post-synaptic Compartments}

\author{
Pragya Goel, Samantha Nishimura, Karthik Chetlapalli, Xiling Li, Catherine Chen \\ and Dion Dickman ${ }^{\star}$
}

Department of Neurobiology, University of Southern California, Los Angeles, CA, United States

Neurons must establish and stabilize connections made with diverse targets, each with distinct demands and functional characteristics. At Drosophila neuromuscular junctions (NMJs), synaptic strength remains stable in a manipulation that simultaneously induces hypo-innervation on one target and hyper-innervation on the other. However, the expression mechanisms that achieve this exquisite target-specific homeostatic control remain enigmatic. Here, we identify the distinct target-specific homeostatic expression mechanisms. On the hypo-innervated target, an increase in postsynaptic glutamate receptor (GluR) abundance is sufficient to compensate for reduced innervation, without any apparent presynaptic adaptations. In contrast, a target-specific reduction in presynaptic neurotransmitter release probability is reflected by a decrease in active zone components restricted to terminals of hyper-innervated targets. Finally, loss of postsynaptic GluRs on one target induces a compartmentalized, homeostatic enhancement of presynaptic neurotransmitter release called presynaptic homeostatic potentiation (PHP) that can be precisely balanced with the adaptations required for both hypo- and hyper-innervation to maintain stable synaptic strength. Thus, distinct anterograde and retrograde signaling systems operate at pre- and post-synaptic compartments to enable target-specific, homeostatic control of neurotransmission.

Keywords: active zone, homeostasis, synaptic plasticity, Drosophila, neuromuscular junction

\section{INTRODUCTION}

Synapses are spectacularly diverse in their morphology, architecture, and functional characteristics. These differences are reflected in the molecular composition and abundance of synaptic components at heterogeneous synaptic subtypes in central and peripheral nervous systems (Atwood and Karunanithi, 2002; Branco and Staras, 2009; O'Rourke et al., 2012). Interestingly, the structure and function of synapses can also vary substantially across terminals of an individual neuron (Guerrero et al., 2005; Grillo et al., 2018; Fekete et al., 2019) and drive input-specific presynaptic plasticity (Letellier et al., 2019). Both Hebbian and homeostatic plasticity mechanisms can work locally and globally at specific synapses to tune synapse function, enabling stable yet flexible ranges of synaptic strength (Turrigiano, 2012; Vitureira and Goda, 2013; Diering and Huganir, 2018). For example, homeostatic receptor scaling globally adjusts glutamate receptor (GluR) 
abundance, subtype, and/or functionality at dendrites (Turrigiano and Nelson, 2004) yet there is also evidence for synapse specificity (Sutton et al., 2006; Hou et al., 2008; Béique et al., 2011). Although studies have begun to elucidate the factors that enable both local and global modes of synaptic plasticity at synaptic compartments, it is less appreciated how and why specific synapses undergo plasticity within the context and needs of information transfer in a neural circuit.

One major force that sculpts the heterogeneity of synaptic strength is imposed through the specific targets being innervated. For example, studies at neuromuscular synapses in the stomatogastric system of lobsters have demonstrated that presynaptic terminals of the same motor axon can concurrently undergo facilitation and depression due to differences in the synapses made onto two postsynaptic muscle fibers (Katz et al., 1993). Furthermore, at vertebrate neuromuscular junctions (NMJs), secreted factors from muscles can dictate which motor neurons survive during development and in many cases their neurotransmitter phenotype (Schotzinger and Landis, 1990; Calderó et al., 1998). Parallel target-dependent control of neuropeptide identity has also been shown in the Drosophila central nervous system (Allan et al., 2003; Allan and Thor, 2015). In mammalian central neurons, factors such as BDNF secreted from postsynaptic dendrites not only promote neuronal survival but also can homeostatically enhance presynaptic neurotransmitter release and functional properties of neural circuits (Jakawich et al., 2010; Park and Poo, 2013), while postsynaptic signaling through $\mathrm{N}$-Cadherins and mTORC1 can regulate presynaptic function (Vitureira et al., 2011; Henry et al., 2012). Finally, at the Drosophila NMJ, presynaptic homeostatic plasticity can be expressed at a subset of terminals within a single motor neuron depending on GluR functionality at particular targets ( $\mathrm{Li}$ et al., 2018a), demonstrating that this form of homeostatic plasticity is target-specific and strongly suggesting it is also synapse-specific. Together, these studies and others have demonstrated that the physiologic, metabolic, and/or structural properties at terminals of a single neuron can be selectively modulated according to the identity and needs of the targets they innervate. However, the nature of the transsynaptic dialogue and the molecular mechanisms that achieve target-specific plasticity are not well understood.

A seminal study published over 20 years ago found that distinct target-specific modulations in synaptic activity maintain stable neurotransmission following biased innervation at terminals of motor neurons at the Drosophila NMJ (Davis and Goodman, 1998). In this manipulation, biased innervation is achieved by overexpression of the trans-synaptic cell adhesion factor Fasciculin II (FasII) on one of the two muscle targets innervated by motor neurons (Davis and Goodman, 1998). This leads to hyper-innervation of the target overexpressing FasII at the expense of the adjacent target, which is hypo-innervated. Remarkably, synaptic strength, as assessed by electrophysiological recordings, was maintained at levels similar in amplitude to normally innervated NMJ targets. Since this pioneering study, however, the molecular and cellular expression mechanisms that achieve this target-specific homeostatic modulation have remained enigmatic.
We have investigated how terminals of an individual neuron adapt to simultaneous hypo- and hyper-innervation to maintain stable synaptic strength on two adjacent targets. Our analysis reveals that a novel homeostatic signaling system operates in the hypo-innervated target to precisely enhance the abundance of postsynaptic GluRs, offsetting reduced presynaptic neurotransmitter release and stabilizing synaptic strength. In contrast, no apparent adaptations are observed in the hyper-innervated target. Rather, presynaptic release probability is homeostatically reduced, accompanied by a targetspecific decrease in the abundance and density of active zone components. Finally, we find that presynaptic homeostatic potentiation (PHP) can be selectively induced and expressed at synapses on one target and balanced with biased innervation to sustain stable synaptic strength. This work reveals the striking interplay of target-specific homeostasis modulating the efficacy of neurotransmission across synaptic terminals.

\section{MATERIALS AND METHODS}

\section{Fly Stocks}

Drosophila stocks were raised at $25^{\circ} \mathrm{C}$ on standard molasses food. The $w^{1118}$ strain is used as the wild type control unless otherwise noted as this is the genetic background in which all genotypes are bred. The H94-Gal4 driver, which expresses transiently early in larval development (Davis et al., 1997), was sufficient to induce biased innervation when crossed to UAS-FasII (Davis and Goodman, 1998; used in Figures 1-3). However, this driver alone is not sufficient to knock-down GluRIIA when crossed to UAS-GluRIIA-RNAi ( $\mathrm{Li}$ et al., 2018a). Therefore, the same manipulation developed in ( $\mathrm{Li}$ et al., 2018a) was used for the experiments detailed in Figure 4 and Supplementary Figure S3, where a cassette amplifies and maintains Gal4 expression after transient activation by the H94-Gal4 driver. This results in a persistently strong expression of the UAS-FasII and UASGluRIIA-RNAi transgenes in muscle 6. Details of all stocks and their sources are listed in the Reagents and Resource Supplementary Table S1.

\section{Immunocytochemistry}

Third-instar larvae were dissected in ice-cold $0 \mathrm{Ca}^{2+} \mathrm{HL}-3$ and immunostained using a standard protocol as described (Perry et al., 2017). In brief, larvae were either fixed in Bouin's fixative for $5 \mathrm{~min}$ (Sigma, HT10132-1L), 100\% ice-cold ethanol for $5 \mathrm{~min}$, or $4 \%$ paraformaldehyde (PFA) for $10 \mathrm{~min}$. Larvae were then washed with PBS containing $0.1 \%$ Triton X-100 (PBST) for 30 min, blocked with 5\% Normal Donkey Serum followed by overnight incubation in primary antibodies at $4^{\circ} \mathrm{C}$. Preparations were then washed $3 \times$ in PBST, incubated in secondary antibodies for $2 \mathrm{~h}$, washed $3 \times$ in PBST, and equilibrated in $70 \%$ glycerol. Before imaging, samples were mounted in VectaShield (Vector Laboratories). Details of all antibodies, their source, dilution, and references are listed in Supplementary Table S1.

\section{Confocal Imaging and Analysis}

Samples were imaged using a Nikon A1R Resonant Scanning Confocal microscope equipped with NIS Elements software and 
A

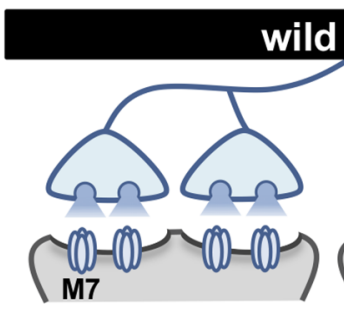

wild type

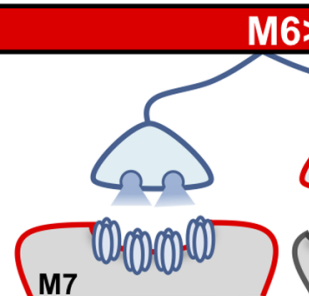

M6>FasIl

B
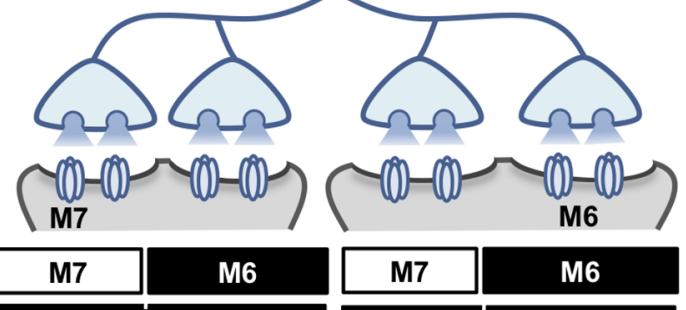

HRP
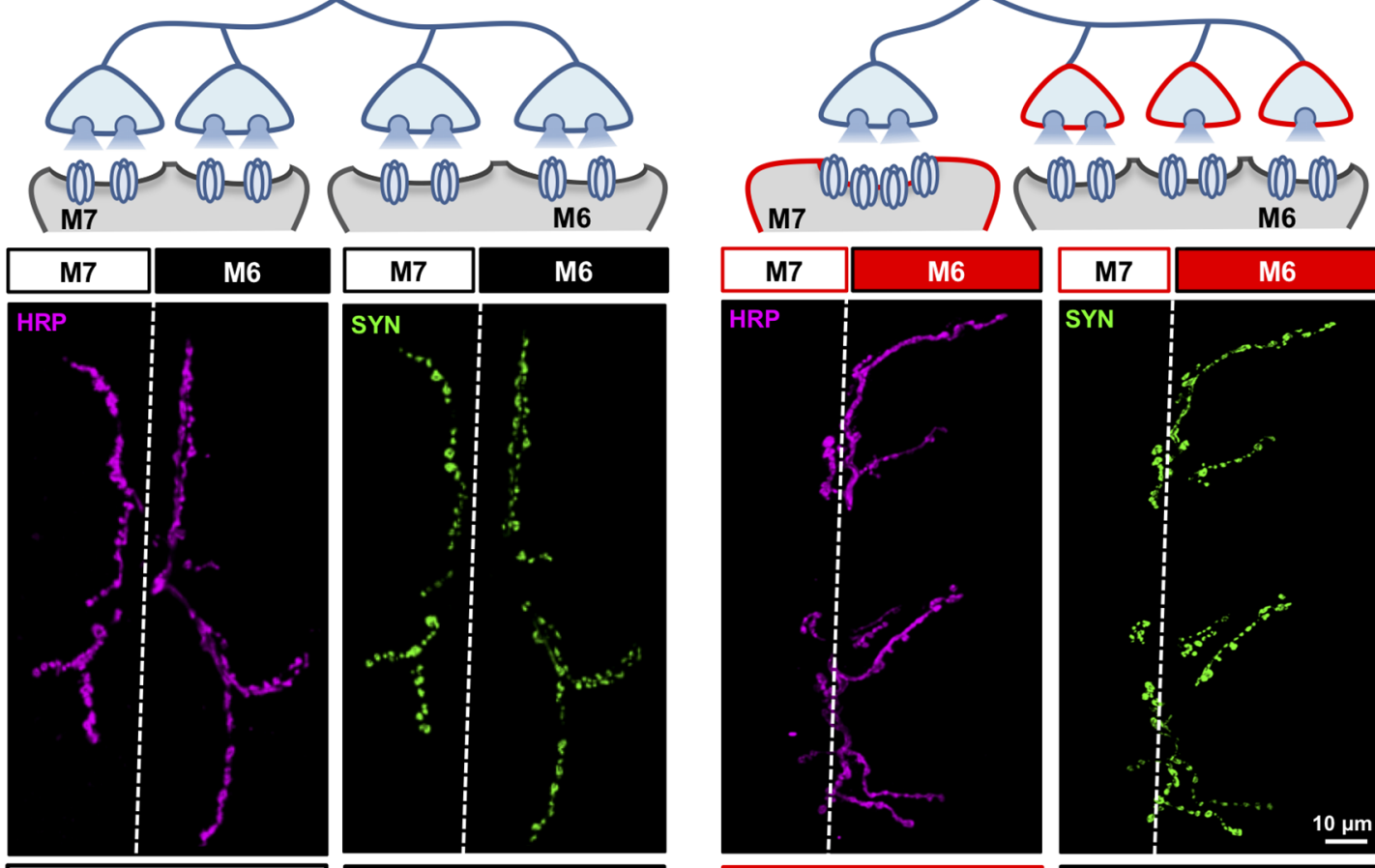

C M7
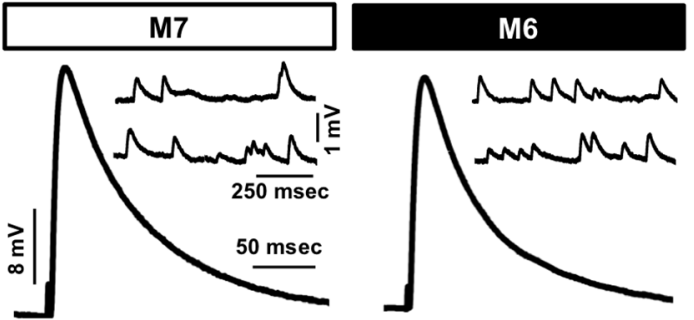

D

muscle 7 (M6>FasII)
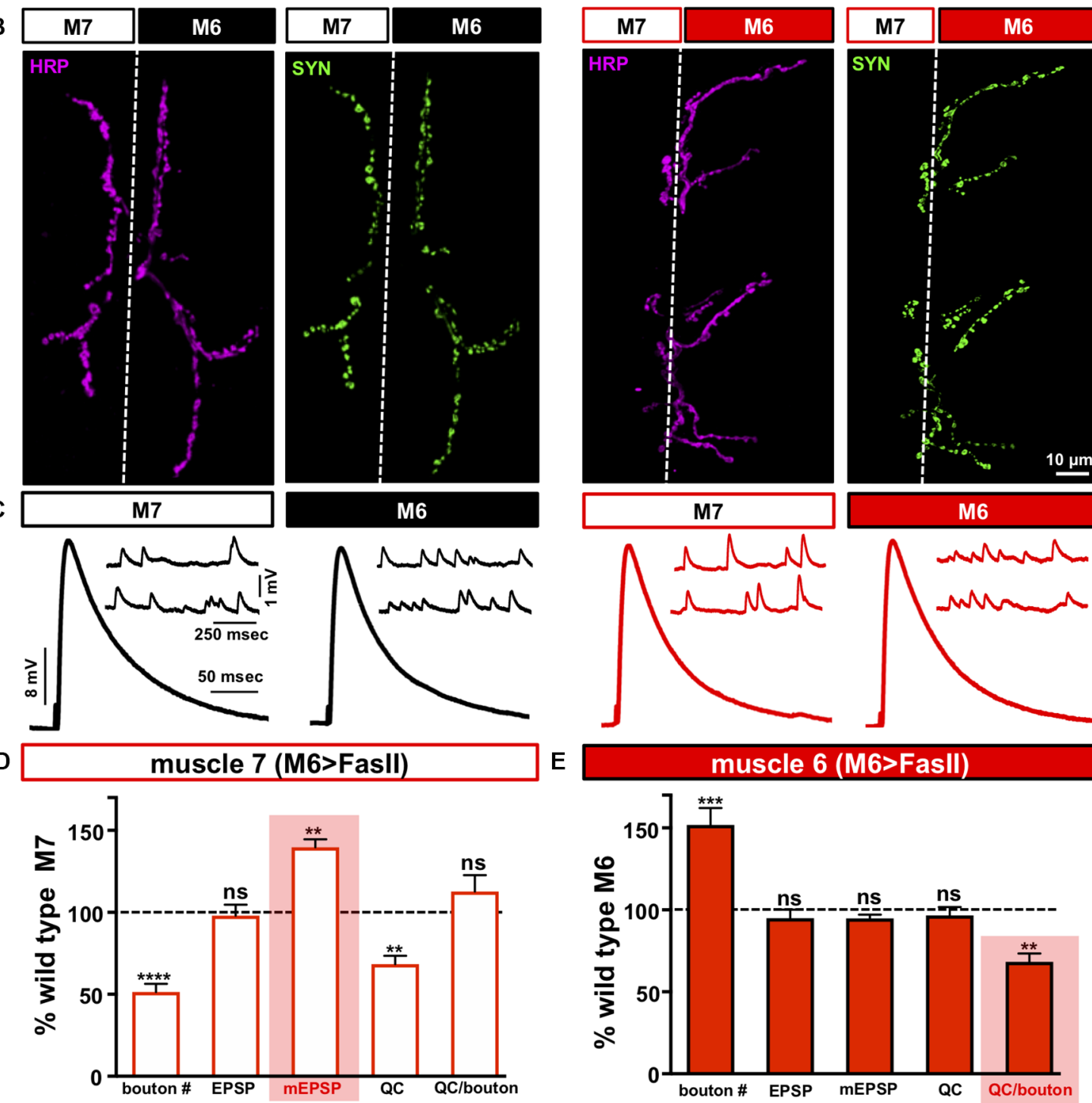

$\mathrm{E}$

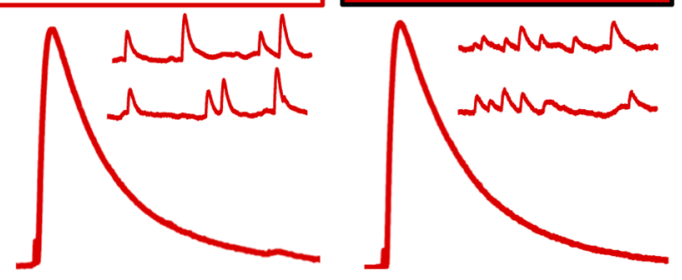

muscle 6 (M6>FasII)

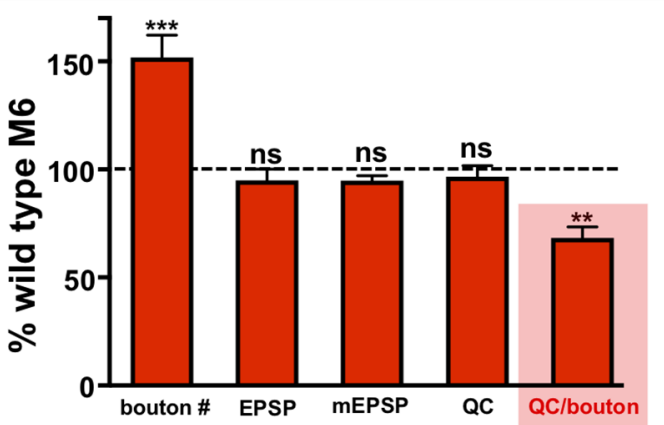

FIGURE 1 | Biased innervation at the neuromuscular junction (NMJ) elicits distinct target-specific homeostatic adaptations. (A) Schematic of a motor neuron innervating both muscle 6 and 7 at the Drosophila larval NMJ. Biased innervation is achieved by overexpressing the cell adhesion factor Fasll specifically on muscle 6 using H94-Gal4 (M6 >Fasll: w; UAS-Fasl//+; H94-Ga/4/+). Red outlines highlight the likely synaptic compartment in which the adaptation occurs.

(B) Representative images of muscle 6/7 neuromuscular junctions (NMJs) immunostained with antibodies that recognize the neuronal membrane (Horshradish Peroxidase; HRP) and synaptic vesicles (Synapsin; SYN) in wild type $\left(w^{1118}\right)$ and M6 $>$ Fasll. Note that while boutons labeled by SYN puncta are roughly equally split between muscles 6 and 7 in wild type, M6 >Fasll causes biased innervation on muscle 6 at the expense of muscle 7. (C) Representative electrophysiological traces of recordings from muscles 7 and 6 in wild type and M6 >Fasll NMJs. Note that while EPSP amplitudes are similar across all muscles, miniature excitatory postsynaptic potentials (mEPSPs) are increased only on muscle 7 of M6 > Fasll. (D) Quantification of bouton number, EPSP amplitude, mEPSP amplitude, quantal content, and quantal content normalized per bouton on muscle 7 in M6 $>$ Fasll. All values are normalized to the values at wild type muscle 7 . Enhanced mEPSP amplitude (shaded bar) implies reduced quantal content and no change in quanta released per bouton. (E) Quantification of all values in (D) on muscle 6 of M6 > Fasll normalized to wild type muscle 6 values. Note that the estimated quantal content per bouton (shaded bar) is significantly reduced. Error bars indicate $\pm \operatorname{SEM}\left(n \geq 16\right.$; one-way ANOVA; Supplementary Table S2). ${ }^{* *} p<0.01 ;{ }^{* \star \star} p<0.001 ;{ }^{* \star \star *} p<0.0001$; ns, not significant. 


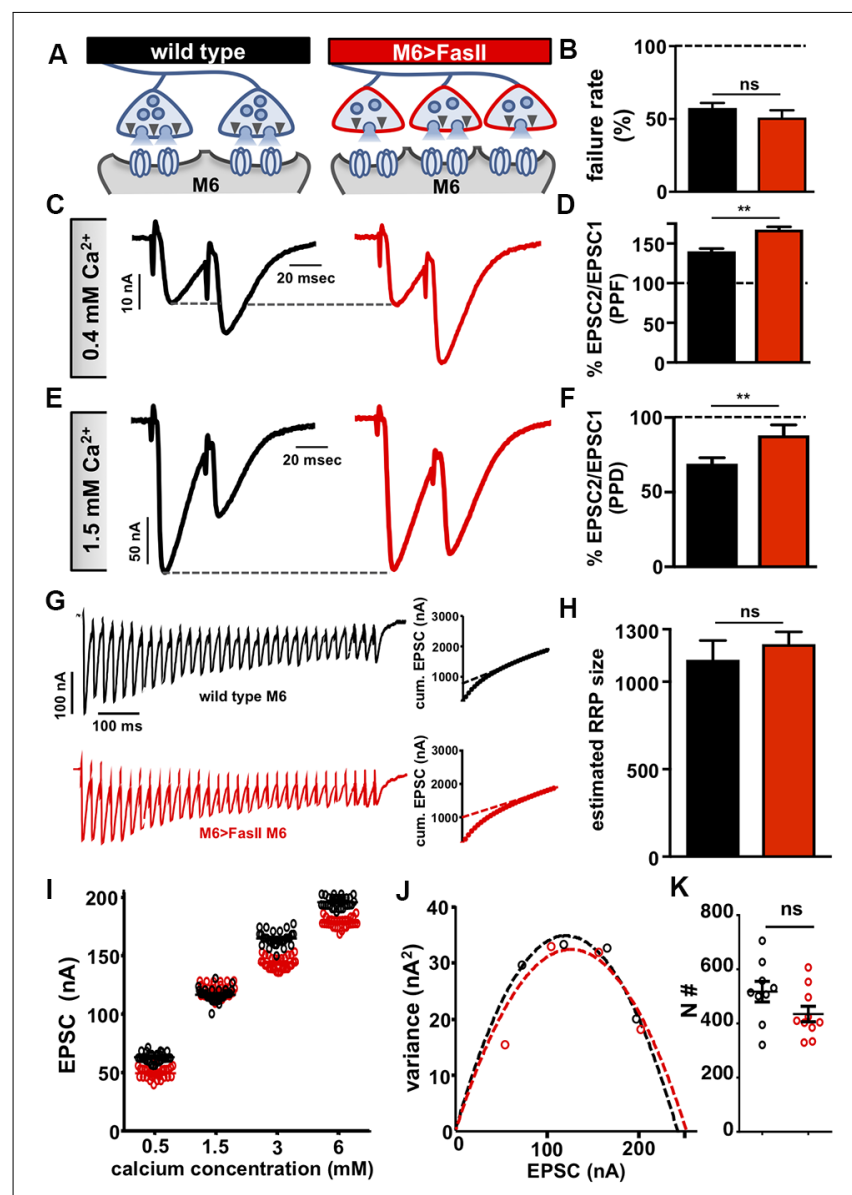

FIGURE 2 | Hyper-innervation elicits a homeostatic decrease in presynaptic release probability. (A) Schematic illustrating a reduction in readily releasable pool (RRP) size and functional release site number on hyper-innervated muscle 6. (B) Failure analysis reveals no significant change in failure rate on muscle 6 in M6 > Fasll, consistent with unaltered quantal content on this target. (C) Representative paired-pulse EPSC traces at $0.4 \mathrm{mM}$ extracellular $\mathrm{Ca}^{2+}$ with an interstimulus interval of $16.7 \mathrm{~ms}$ in the indicated genotypes. Increased paired-pulse facilitation (PPF) was observed on hyper-innervated targets, consistent with reduced release probability. (D) Quantification of PPF ratio (EPSC2/EPSC1). (E) Representative paired-pulse EPSC traces at $1.5 \mathrm{mM}$ extracellular $\mathrm{Ca}^{2+}$ with an interstimulus interval of $16.7 \mathrm{~ms}$ in the indicated genotypes. Reduced paired-pulse depression (PPD) was observed on hyper-innervated targets, consistent with a reduced probability of release. (F) Quantification of PPD ratio (EPSC2/EPSC1) shows an increase. (G) Representative EPSC recordings of 30 stimuli at $3 \mathrm{mM}$ extracellular $\mathrm{Ca}^{2+}$ during a $60 \mathrm{~Hz}$ stimulus train in the indicated genotypes. Insets represent the average cumulative EPSC plotted as a function of time. A-line fit to the $18^{\text {th }}-30^{\text {th }}$ stimuli was back-extrapolated to time 0 . $(\mathbf{H})$ The estimated size of the RRP is unchanged on muscle 6 in M6 > Fasll compared with wild type, suggesting reduced RRP per bouton. (I) Scatter plot EPSC distribution of recordings on muscle 6 from wild type and M6 $>$ Fasll in the indicated extracellular $\mathrm{Ca}^{2+}$ concentrations. (J) Variance-mean plots for the indicated genotypes. Dashed lines are the best-fit parabolas to the data points. (K) The estimated number of functional release sites (N\#) obtained from the variance-mean plots in $\mathbf{( J )}$ showing no significant difference between the genotypes. Error bars indicate \pm SEM ( $n \geq 9$; one-way ANOVA;

Supplementary Table S2). ${ }^{\star \star} p<0.01$; ns, not significant.

a $60 \times$ APO 1.4 NA oil immersion objective using separate channels with four laser lines $(405,488,561$, and $637 \mathrm{~nm})$ at room temperature. Boutons were counted using NMJs stained with anti-Synapsin or -vGlut, co-stained with anti-HRP on muscle 6/7 of segment A2 and A3, considering each Synapsin or vGlut punctum to be a bouton. For fluorescence quantifications of postsynaptic GluRs and active zone proteins, all genotypes within a data set were immunostained in the same tube with identical reagents, then mounted and imaged in the same session. Z-stacks were obtained using identical settings for all genotypes with $\mathrm{Z}$-axis spacing between $0.15-0.2 \mu \mathrm{m}$ within an experiment and optimized for detection without saturation of the signal. Maximum intensity projections were used for quantitative image analysis with the NIS Elements General Analysis toolkit.

To quantify the sum punctum intensity, the total fluorescence intensity signal of each punctum was calculated without regard to the area as described (Goel et al., 2019a). For each particular sample set, thresholds were optimized to capture the dynamic range of intensity levels within the wild type sample. This same threshold was then used to image all other genotypes in the sample set, and all intensities were normalized to wild type values within an experimental set. Active zones too closely spaced to be resolved ( $\sim 5 \%$ of all analyzed) were excluded from the analysis. Spot detection in the Nikon Elements Software was used to identify individual BRP and Cac puncta as it resolves closely spaced puncta more accurately compared to thresholding. Finally, to quantify total intensity per NMJ, the fluorescence intensity for each punctum (sum intensity) was added together across the entire NMJ. For calculation of BRP and $\mathrm{Cac}$ puncta density, the total number of puncta at a particular muscle was divided by the neuronal membrane area labeled by HRP spanning that muscle (Goel et al., 2019a). For image representation only, the gain and contrast were adjusted identically for all genotypes within a dataset. To show representative images of individual boutons, a particular area was selected from the entire NMJ (denoted with a white box) and rotated and cropped to demonstrate changes at boutons more clearly.

\section{Electrophysiology}

All dissections and recordings were performed in modified HL-3 saline (Stewart et al., 1994; Kikuma et al., 2017) containing (in mM): $70 \mathrm{NaCl}, 5 \mathrm{KCl}, 10 \mathrm{MgCl}_{2}, 10 \mathrm{NaHCO}_{3}$, 115 Sucrose, 5 Trehalose, 5 HEPES, and $0.4 \mathrm{CaCl}_{2}$ (unless otherwise specified), $\mathrm{pH}$ 7.2. Neuromuscular junction sharp electrode (electrode resistance between 10-35 M $\Omega$ ) recordings were performed on muscles 6 and 7 of abdominal segments A2 and A3 in wandering third-instar larvae (Kiragasi et al., 2017). Recordings were performed on an Olympus BX61 WI microscope using a $40 \times / 0.80$ NA water-dipping objective. Recordings were acquired using an Axoclamp 900A amplifier, Digidata 1440 A acquisition system, and pClamp 10.5 software (Molecular Devices). Electrophysiological sweeps were digitized at $10 \mathrm{kHz}$ and filtered at $1 \mathrm{kHz}$. Data were analyzed using Clampfit (Molecular devices), MiniAnalysis (Synaptosoft), and Excel (Microsoft) software.

Miniature excitatory postsynaptic potentials (mEPSPs) were recorded in the absence of any stimulation and cut motor axons were stimulated to elicit excitatory postsynaptic potentials 
A

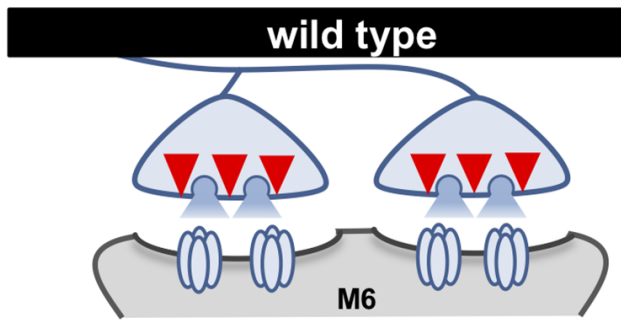

B

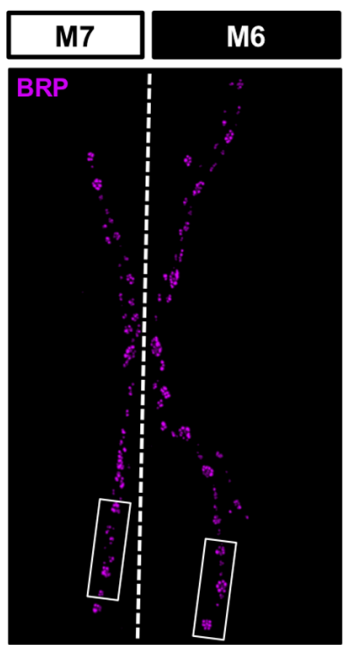

C

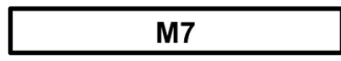

$\frac{n}{n}$

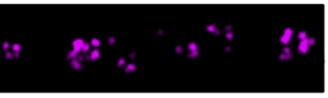

䂤
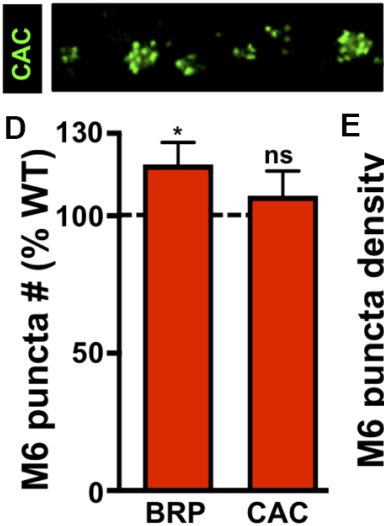
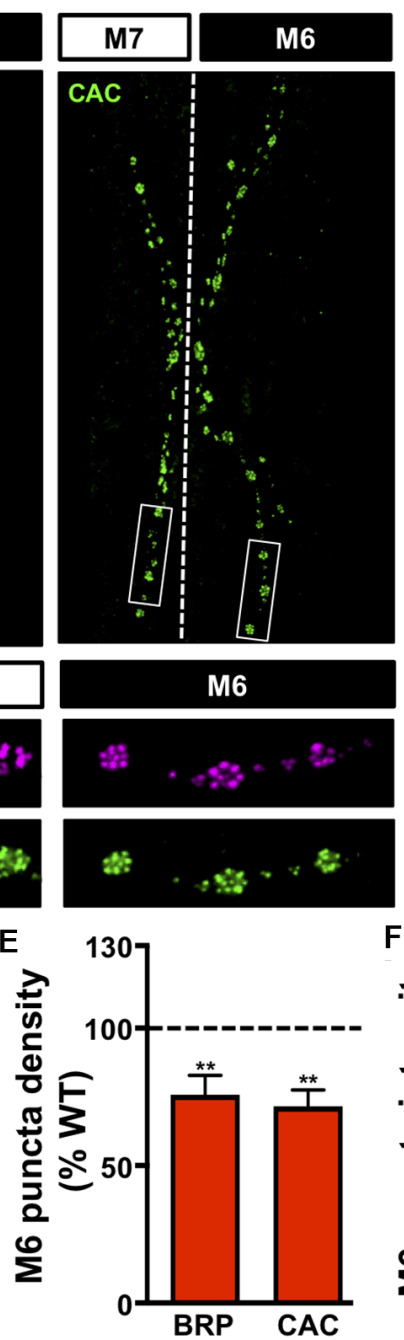
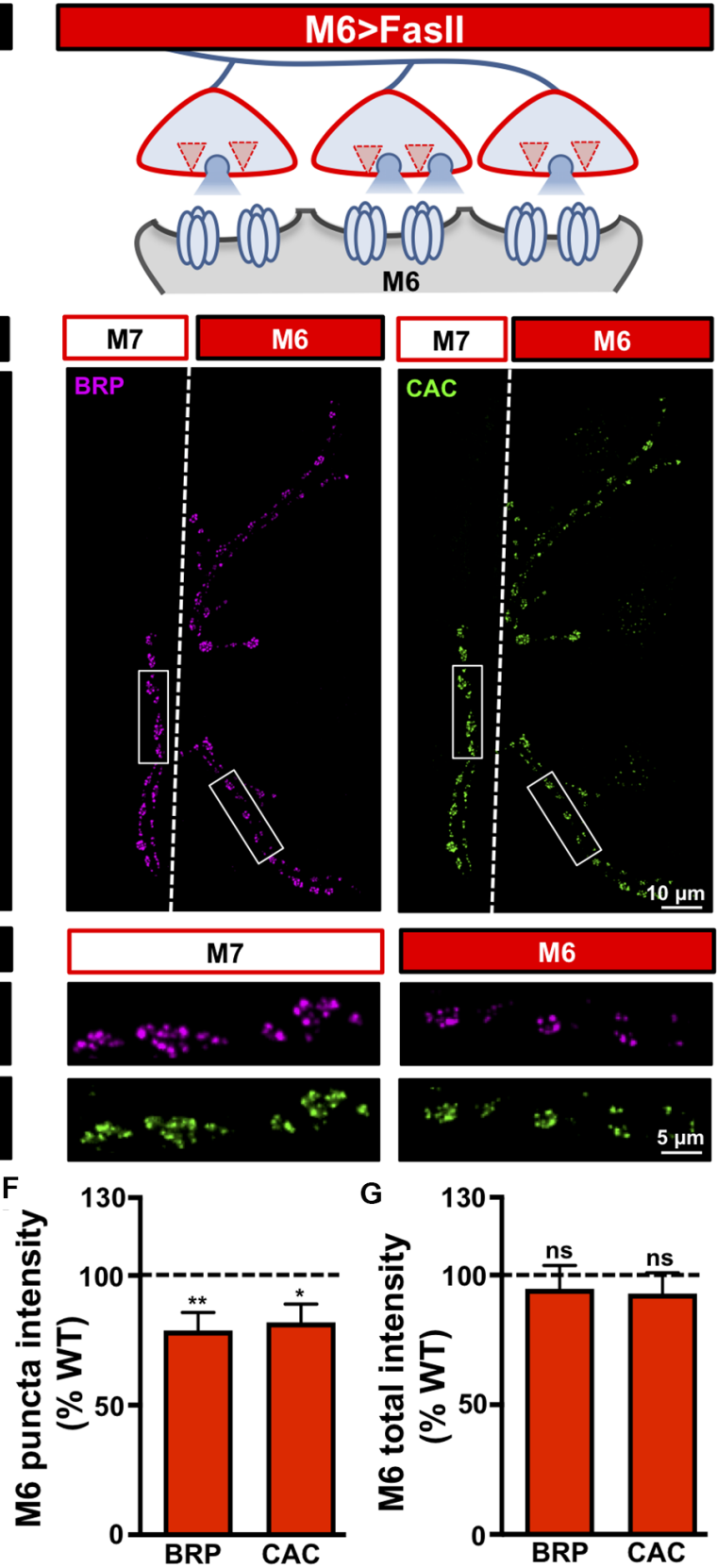

FIGURE 3 | Target-specific reductions in both active zone density and intensity at hyper-innervated NMJs. (A) Schematic illustrating a reduction in the number and intensity of active zones at individual boutons on hyper-innervated muscle 6. (B) Representative images of muscle 6/7 NMJs in the indicated genotypes (wild type: cac $^{\text {sfGFP-N }}$; M6 > Fasll: cac ${ }^{\text {sfGFP-N }}$; UAS-Fasll/+; H94-Gal4/+) immunostained with antibodies against the active zone scaffold bruchpilot (BRP) and GFP to label endogenously tagged $\mathrm{Ca}^{2+}$ channels (CAC). (C) Individual boutons from selected areas (white rectangles) of NMJs stained with BRP and CAC in the indicated genotypes and muscles. Note the reduction in the number and intensity of BRP and CAC puncta specifically on muscle 6 in M6 >Fasll, while no change is observed on muscle 7 relative to wild type controls. Quantification of BRP and CAC puncta number (D) and density (E) on muscle 6 in M6 $>$ Fas II normalized as a percentage of wild type muscle 6 values reveals a small but significant increase in BRP puncta number, while BRP and CAC puncta density is significantly reduced on muscle 6 in M6 > Fasll. Quantification of BRP and CAC intensity (F) shows a significant reduction in muscle 6 in M6 $>$ Fasll, while the total fluorescence intensity of all BRP and CAC puncta summed across the entire muscle $6 \mathrm{NMJ}(\mathbf{G})$ is unchanged compared to wild type muscle 6 . Error bars indicate \pm SEM ( $n \geq 13$; one-way ANOVA; Supplementary Table S2). ${ }^{*} p<0.05$; ${ }^{* *} p<0.01$; ns, not significant.

(EPSPs). An ISO-Flex stimulus isolator (A.M.P.I.) was used to modulate the amplitude of stimulatory currents. The intensity was adjusted for each cell, set to consistently elicit responses from both neurons innervating the muscle segment, but avoiding overstimulation. Average mEPSP, EPSP, and quantal content were calculated for each genotype. Muscle input resistance 
A

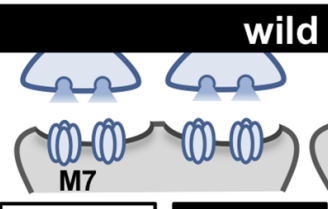

B
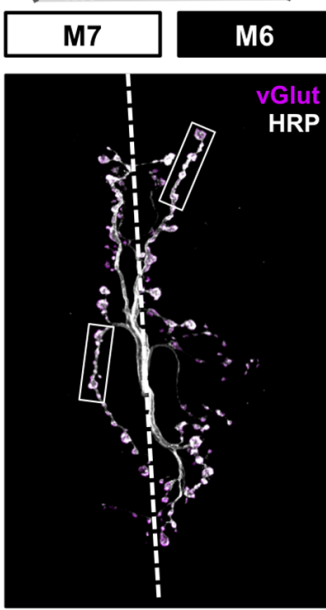

C

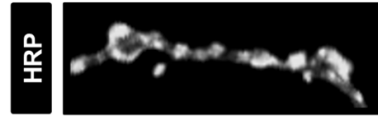

高

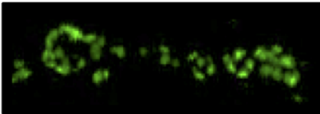

D

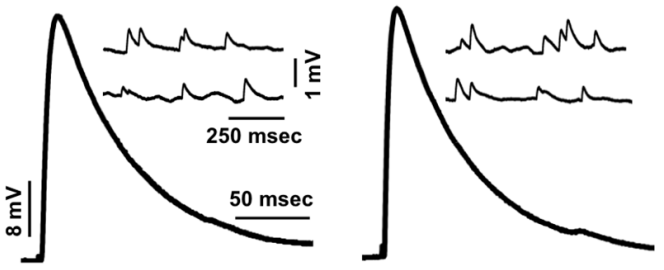

E

muscle 7 (M6>FasII+GluRIIA ${ }^{\text {RNAi }}$

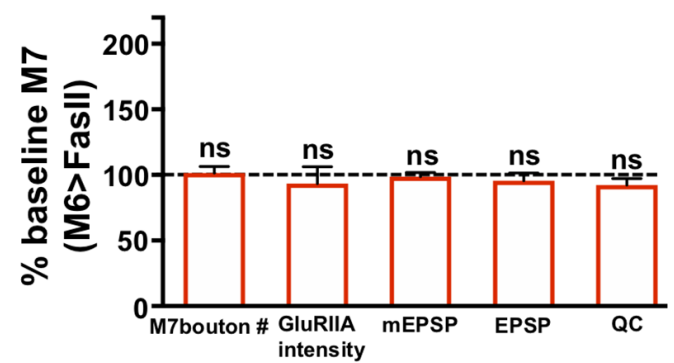

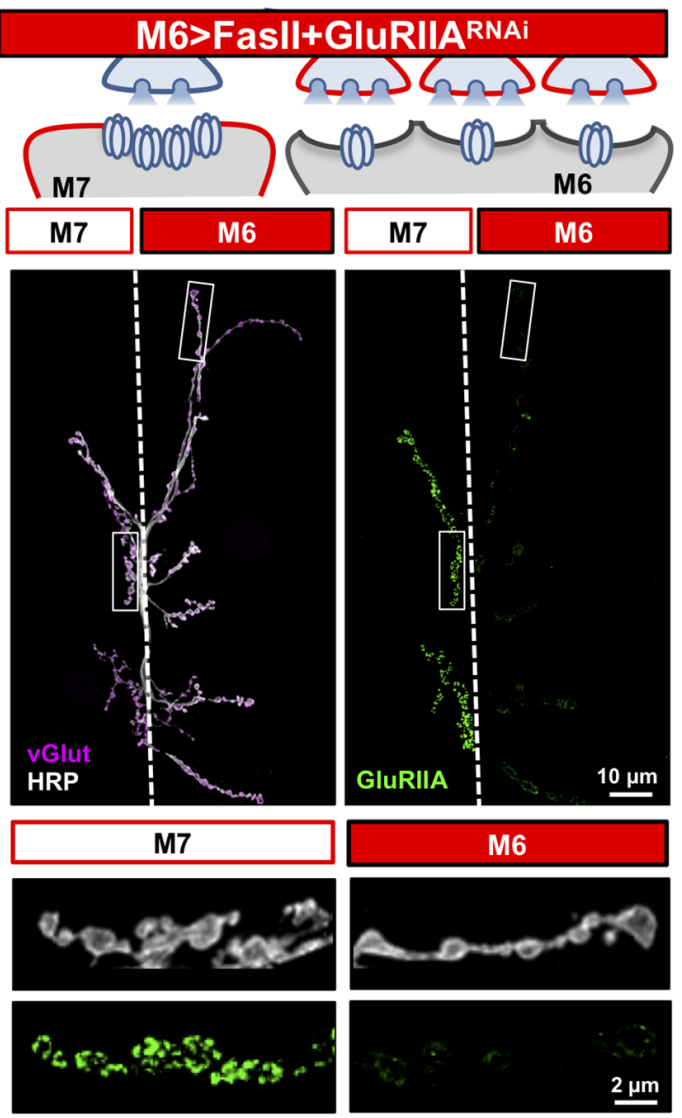
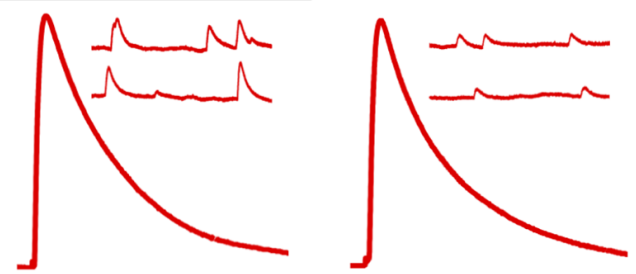

$\mathbf{F}$

muscle 6 (M6>FasIl+GluRIIA RNAi)

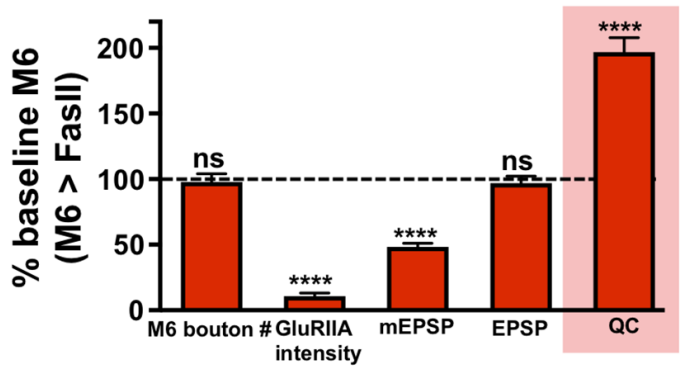

FIGURE 4 | Distinct target-specific adaptations balance hyper-innervation and GluR loss. (A) Schematic illustrating the dual manipulation used to both bias innervation and inhibit GluRIIA expression specifically on muscle 6 (M6 >Fasll+GluRIIARAi: w; Tub-FRT-STOP-FRT-Gal4, UAS-FLP, UAS-CD8-GFP; H94-Gal4, nSyb-Gal80/UAS-Fasll; UAS-G/uRIIARAi). (B) Representative images of muscle 6/7 NMJs from the indicated genotypes immunostained with anti-HRP, -vGlut, and -GluRIIA. (C) Individual boutons from selected areas (white rectangles) of NMJs shown in (B). Note the enhanced GluRIIA levels on hypo-innervated muscle 7 with a loss on hyper-innervated muscle 6. (D) Electrophysiological traces of recordings from muscles 7 and 6 in the indicated genotypes. EPSP amplitudes on muscle 7 and 6 in M6 >FasII+GluRIIARAi are similar to wild-type values. (E) Quantification of bouton numbers, GluR\|A puncta intensity, mEPSP amplitude, EPSP amplitude, and quantal content on muscle 7 in M6 > Fasll+GluRIIARNAi. All values are normalized to baseline (M6 > Fasll muscle 7); no significant differences are observed. (F) Quantification of all values in (D) on muscle 6 of M6 > Fasll+GluRIIA RNAi normalized to baseline (M6 > Fas II muscle 6) values. Note that while GluRIIA levels and mEPSP amplitudes are significantly reduced, EPSP amplitude remains unchanged because of a homeostatic increase in quantal content, indicating presynaptic homeostatic potentiation (PHP) expression. Error bars indicate \pm SEM ( $n \geq 8$; Student's $t$-test; Supplementary Table S2). ${ }^{\star \star \star *} p<0.0001$; ns, not significant. 
$\left(\mathrm{R}_{\text {in }}\right)$ and resting membrane potential $\left(\mathrm{V}_{\text {rest }}\right)$ were monitored during each experiment. Recordings were rejected if the $V_{\text {rest }}$ was more depolarized than $-60 \mathrm{mV}$, if the $R_{\text {in }}$ was less than $5 \mathrm{M} \Omega$, or if either measurement deviated by more than $10 \%$ during the experiment. Larvae were incubated with or without philanthotoxin-433 (PhTx; Sigma; $20 \mu \mathrm{M}$ ) and resuspended in HL-3 for $10 \mathrm{~min}$ as described (Frank et al., 2006; Dickman and Davis, 2009).

The readily releasable pool (RRP) size was estimated by analyzing cumulative EPSC amplitudes while recording in a two-electrode voltage clamp (TEVC) configuration as described (Goel et al., 2019c). Muscles were clamped at $-80 \mathrm{mV}$ and EPSCs were evoked with a $60 \mathrm{~Hz}, 60$ stimulus train while recording in $3 \mathrm{mM} \mathrm{Ca}^{2+} \mathrm{HL}-3$. A-line fit to the linear phase (stimuli \#18-30) of the cumulative EPSC data was back-extrapolated to time 0 . The RRP value was estimated by determining the extrapolated EPSC value at time 0 and dividing this value by the average mEPSC amplitude.

Data used in the variance-mean plot was obtained from TEVC recordings using an initial $0.5 \mathrm{mM} \mathrm{Ca}^{2+}$ concentration, which was subsequently increased to $1.5,3.0$, and $6.0 \mathrm{mM}$ through saline exchange using a peristaltic pump (Langer Instruments, BT1002J). EPSC amplitudes were monitored during the exchange, and 30 EPSC $(0.5 \mathrm{~Hz}$ stimulation rate) events were performed in each calcium condition ( $\mathrm{Li}$ et al., 2018a). To obtain the variance-mean plot, the variance (squared standard deviation) and mean (averaged evoked amplitude) were calculated from the 30 EPSCs at each $\mathrm{Ca}^{2+}$ concentration. The variance was then plotted against the mean for each specific calcium condition using MATLAB software (MathWorks, USA). One additional data point, in which variance and mean are both theoretically at 0 , was used for $\mathrm{Ca}^{2+}$-free saline. Data from these five conditions were fit with a standard parabola (variance $=Q^{*} I_{m}-I_{m 2} / N$ ), where $\mathrm{Q}$ is the quantal size, $\mathrm{I}_{\mathrm{m}}$ is the mean evoked amplitude ( $\mathrm{x}$-axis), and $\mathrm{N}$ is the number of functional release sites. $\mathrm{N}$, as a parameter of the standard parabola, was directly calculated for each cell by best parabolic fit.

Failure analysis was performed in an HL-3 solution containing $0.15 \mathrm{mM} \mathrm{CaCl} 2$. At this extracellular $\mathrm{Ca}^{2+}$ concentration, approximately half of the stimulations evoked responses in the muscle in wild type larvae. A total of 40 trials (stimulations) were performed at each NMJ in all genotypes. The failure rate was obtained by dividing the total number of failures by the total number of trials (40). Paired-pulse recordings were performed at a $\mathrm{Ca}^{2+}$ concentration of $0.3 \mathrm{mM}$ to assay facilitation (PPF) and $1.5 \mathrm{mM}$ for depression (PPD). Following the first stimulation, a second EPSC was evoked at an interstimulus interval of $16.66 \mathrm{~ms}$. Paired-pulse ratios were calculated as the difference between the second peak and the maximum value between both peaks (corresponding to the starting point of the second response) divided by the first amplitude.

\section{Statistical Analysis}

Data were analyzed using GraphPad Prism (version 7.0) or Microsoft Excel software (version 16.22). Sample values were tested for normality using the D'Agostino and Pearson omnibus normality test which determined that the assumption of normality of the sample distribution was not violated. Data were then compared using either a one-way ANOVA and tested for significance using a Tukey's multiple comparison test or using an unpaired 2-tailed Student's $t$-test with Welch's correction. All data are presented as mean $\pm \mathrm{SEM} ; n$ indicates sample number and $p$ denotes the level of significance assessed as $\left.\left.p<0.05^{(*)}, p<0.01^{(* *}\right), p<0.0011^{(* *}\right), p<0.0001^{(* * *)}$; ns $=$ not significant. Statistics of all experiments are summarized in Supplementary Table S2.

\section{RESULTS}

\section{Target-Specific Mechanisms Maintain Stable Synaptic Strength at Hypo- and Hyper-innervated NMJs}

We first sought to reproduce and confirm the biased innervation and synaptic electrophysiology reported in Davis and Goodman, 1998. At Drosophila larval NMJs, motor neurons distribute their synaptic terminals roughly evenly between two distinct targets - as demonstrated by the NMJs made onto muscles 6 and 7 (Figure 1A; left). This stereotyped pattern of innervation can be visualized by immunostaining the NMJ with antibodies that recognize the neuronal membrane (HRP) and synaptic vesicles (Synapsin; SYN), which demonstrates $\sim 60 \%$ boutons on the larger muscle 6 and $\sim 40 \%$ on the smaller muscle 7 (Figure 1B; left and Supplementary Table S2). To bias innervation on these targets, we used the H94-Gal4 driver to drive expression of the cell adhesion molecule Fasciculin II (FasII) early in development selectively on muscle 6 (M6 > FasII; Davis and Goodman, 1998). Immunostaining of M6 > FasII NMJs confirmed biased innervation with $\sim 150 \%$ of boutons above controls on muscle 6 (hyper-innervated), and a parallel reduction of $\sim 50 \%$ in boutons on muscle 7 (hypo-innervated) (Figures 1A-E), consistent with the previous study (Davis and Goodman, 1998). However, despite these opposing changes in bouton numbers, electrophysiological recordings of M6 > FasII found that synaptic strength, measured by the excitatory postsynaptic potential (EPSP) amplitude, was similar on both targets and unchanged from their respective controls (Figures 1C-E). This implies target-specific mechanisms modulate neurotransmission on hypo- and hyper-innervated terminals to maintain stable NMJ strength.

To gain insight into how EPSP amplitudes remain similar to baseline values at NMJs with biased innervation, we next examined miniature neurotransmission. On hypo-innervated muscle 7, mEPSP amplitudes were significantly increased by $\sim 40 \%$ compared to baseline values (Figures 1C,D), as previously observed (Davis and Goodman, 1998). Quantal content (QC) was thus decreased by $\sim 40 \%$, a value similar in magnitude to the reduction in bouton number (Figure 1D). In contrast, mEPSP amplitude was not significantly different on the hyper-innervated muscle $6 \mathrm{NMJ}$ compared to baseline (Figures 1C,E), with no apparent change in quantal content (Figure 1E), as previously observed (Davis and Goodman, 1998). Finally, analysis of quantal content normalized per bouton on muscle 6 NMJs revealed an $\sim 30 \%$ reduction (Figure 1E), 
suggesting a target-specific, homeostatic decrease in presynaptic neurotransmitter release, consistent with the results of single bouton recordings (Davis and Goodman, 1998). Together, this data indicates that distinct target-specific mechanisms operate to stabilize neurotransmission at hypo- vs. hyper-innervated NMJs.

\section{A Homeostatic Increase in Postsynaptic GluR Abundance Stabilizes Synaptic Strength on Hypo-innervated Targets}

It was previously reported that at hypo-innervated NMJs following M6 > FasII, levels of the postsynaptic GluR subunit GluRIIA were increased (Goel and Dickman, 2018). We, therefore, focused on postsynaptic adaptations to GluRs, as we considered two possible presynaptic changes unlikely. First, increased presynaptic vesicle size could in principle lead to enhanced glutamate emitted per vesicle, as has been documented in endocytosis mutants and following overexpression of the vesicular glutamate transporter (Daniels et al., 2004; Goel et al., 2019a). However, there is no evidence for endocytic defects or increased $v$ Glut expression induced by the M6 > FasII manipulation. Second, although multivesicular release has been observed in some systems (Rudolph et al., 2015) and was raised as a possibility in the original study to potentially explain the increased quantal size (Davis and Goodman, 1998), multi-vesicular release at the fly NMJ is rarely if ever observed (Melom et al., 2013; Brusich et al., 2018). Hence, we focused on possible postsynaptic mechanisms to explain the increased mEPSP amplitude on hypo-innervated NMJs, which may parallel the ones that have been documented in mammalian forms of homeostatic receptor scaling (Turrigiano, 2008; Diering and Huganir, 2018). These include increases in the abundance, subtype, and/or functionality of additional postsynaptic GluRs, including GluRIIB-containing receptors, as enhanced levels of GluRIIA-containing GluRs were recently reported at hypo-innervated NMJs in Drosophila (Goel and Dickman, 2018; Goel et al., 2019b).

We, therefore, examined postsynaptic GluR levels in hypo-innervated targets induced by M6 >FasII. At the Drosophila NMJ, the postsynaptic response to glutamate is mediated by two subtypes of GluRs, GluRIIA- and GluRIIBcontaining receptors. Both subtypes are composed of the essential subunits GluRIIC, GluRIID, and GluRIIE but differ in containing either GluRIIA or GluRIIB subunits (Qin et al., 2005; DiAntonio, 2006). We immunostained hypo-innervated NMJs using antibodies against GluRIIA, GluRIIB, and the common GluRIID subunits and observed an $\sim 45 \%$ decrease in the number of GluR puncta compared to wild type muscle 7 (Supplementary Figures S1A,C), reflecting reduced innervation. However, we found an increase in the intensity of all GluR subunits in hypo-innervated NMJs compared to wild type muscle 7 (Supplementary Figures S1B,D). In principle, the $\sim 55 \%$ increase in GluR abundance is sufficient to explain the increased quantal size and to offset the $\sim 40 \%$ reduction in quantal content to homeostatically maintain stable synaptic strength despite reduced innervation. Consistent with this, we observed no adaptations in the anatomical or functional number of release sites, nor in the size of the RRP (Supplementary Figure S2). These lines of evidence indicate that presynaptic terminals of hypo-innervated NMJs function similarly to wild type, with presynaptic neurotransmitter release onto the muscle 7 NMJ simply reduced by $40 \%$. Thus, a $\sim 55 \%$ increase in postsynaptic GluR abundance per receptor field is sufficient to maintain synaptic strength at hypo-innervated NMJs without reason to invoke other homeostatic adaptations.

\section{Hyper-innervation Induces a Homeostatic Decrease in Presynaptic Release Probability}

We next sought to characterize the expression mechanism that enables stable neurotransmitter output on the hyper-innervated target. It was previously demonstrated that a homeostatic reduction in presynaptic release probability was expressed at hyper-innervated NMJs, where single bouton recordings measured a lower release probability for individual boutons (Davis and Goodman, 1998). Consistent with this conclusion, and in contrast to the adjacent hypo-innervated NMJs, we did not observe any significant changes in postsynaptic GluR levels (Supplementary Figures S1E-H). We next performed a series of electrophysiological assays to probe presynaptic function on the hyper-innervated NMJ. First, we used failure analysis to assess presynaptic release independently of miniature transmission by measuring the number of failed release events in very low extracellular $\mathrm{Ca}^{2+}$ concentrations $(0.15 \mathrm{mM}$; see "Materials and Methods" section). We observed no significant difference in the failure rates on hyper-innervated NMJs compared to wild type (Figure 2B), consistent with overall quantal content being unchanged at these NMJs. Next, we probed short term plasticity by determining paired-pulse ratios in moderate and high extracellular $\mathrm{Ca}^{2+}$. At $0.4 \mathrm{mM} \mathrm{Ca}^{2+}$, we observed an increase in paired-pulse facilitation (PPF) at hyper-innervated NMJs compared to wild type (Figures 2C,D), while at $1.5 \mathrm{mM}$ $\mathrm{Ca}^{2+}$, paired-pulse depression (PPD) was reduced at hyperinnervated NMJs (Figures 2E,F). Since short term facilitation and depression vary inversely with release probability, enhanced PPF, and reduced PPD are indicative of reduced release probability (Regehr, 2012). While overall release probability, as calculated by failure analysis, is unchanged at hyper-innervated NMJs, the PPF/PPD findings may reflect altered short term $\mathrm{Ca}^{2+}$ and/or vesicle dynamics at individual release sites. Indeed, an inverse effect on short-term facilitation was reported in rab3 mutants, which have reduced number but enhanced size of active zones (Graf et al., 2009). These results suggest that a targetspecific, homeostatic decrease in presynaptic release probability at individual release sites serves to stabilize transmission at hyper-innervated NMJs.

Although the PPF/PPD recordings suggested reduced release probability at individual active zones of hyper-innervated terminals, the magnitude of the observed decrease $(\sim 25 \%)$ was not sufficient to fully compensate for the $\sim 50 \%$ increase in bouton numbers. We found no change in the size of the RRP on hyper-innervated NMJs compared to wild type (Figures $\mathbf{2 G}, \mathbf{H}$ ), suggesting that the size of the RRP at individual boutons might 
be reduced on muscle 6 of M6 > FasII NMJs. Finally, no change in the total number of functional release sites was observed on hyper-innervated targets (Figures $\mathbf{2} \mathbf{I}-\mathbf{K}$ ), indicating a reduction in the number of release sites participating in neurotransmission per bouton at hyper-innervated NMJs. Thus, a homeostatic adjustment in the release probability of individual active zones and the number of release sites per bouton selectively modulate transmission at hyper-innervated NMJs without measurably impacting release at adjacent hypo-innervated terminals.

\section{A Target-Specific Reduction in Both Active Zone Density and Intensity Is Observed at Hyper-innervated NMJs}

Our electrophysiological data above suggests a reduction in both release probability and the number of functional release sites at individual boutons of hyper-innervated NMJs. In principle, a target-specific reduction in the number and/or function of anatomical release sites could explain these electrophysiological properties. Also, recent evidence indicates that bi-directional changes in the size and nano-structure of active zone architecture at Drosophila NMJs can adjust release probability at individual active zones (Akbergenova et al., 2018; Böhme et al., 2019; Goel et al., 2019a; Gratz et al., 2019). We, therefore, characterized the number and intensity of individual active zones on hyper-innervated NMJs by immunostaining the central scaffold BRP and endogenously tagged CaV2.1 calcium channels (Cac ${ }^{\text {sfGFP }}$; Gratz et al., 2019), defining each BRP punctum to be an active zone. Interestingly, while a $\sim 55 \%$ increase in bouton number was observed at hyper-innervated NMJs, the number of active zones was only increased by $\sim 20 \%$, reflected in a concomitant decrease in active zone density (Figures 3A-E). Thus, hyper-innervated NMJs exhibit a target-specific reduction in the density of release sites that is sufficient in magnitude to limit the increase in active zones to only about $20 \%$ despite an $~ 50 \%$ increase in innervation.

We also quantified the intensity of individual BRP puncta on hyper-innervated NMJs and observed an 20\% decrease in the sum intensity of individual BRP puncta compared to wild type (Figures 3B,C,F). Similar results for puncta density and intensity were found for Cac ${ }^{\text {sfGFP }}$ (Figures 3B-F). Finally, given these reductions in the density and intensity of active zone components, the total intensity of both BRP and Cac ${ }^{\text {sfGFP }}$ per hyper-innervated NMJ was not significantly different from wild type despite the increase in their total number (Figure 3G). These results parallel recent studies that have shown that while the number and intensity of individual active zones can vary at NMJs, the total abundance of active zone protein remains constant (Graf et al., 2009; Goel et al., 2019a,b) or can reflect nanoscale remodeling of active zone components (Böhme et al., 2019; Mrestani et al., 2020). Together, hyperinnervated NMJs express a target-specific reduction in both the number and intensity of release sites per bouton and a parallel reduction in presynaptic release probability that stabilizes synaptic strength, while no reciprocal changes are observed at hypo-innervated counterparts.

\section{Distinct Target-Specific Adaptations Can Homeostatically Balance Hyper-innervation and GluR Perturbation}

When biased innervation of the NMJ is induced through M6>FasII, the hypo-innervated target responds by homeostatically increasing GluR abundance, while the subset of motor neuron terminals that hyper-innervate the adjacent target selectively reduce the number and apparent abundance of active zone components. In our final set of experiments, we sought to determine whether the target-specific homeostatic adaptations triggered by biased innervation could be balanced with an additional target-specific homeostatic challenge. PHP is a well-studied form of homeostatic plasticity at the Drosophila NMJ. Here, rapid pharmacological or chronic genetic manipulations that diminish postsynaptic GluR functionality trigger a trans-synaptic retrograde signaling system that homeostatically increases presynaptic glutamate release to maintain stable synaptic strength (Frank et al., 2020). Recently, it was demonstrated that GluR knockdown specifically on muscle 6 can trigger PHP selectively at the subset of synapses innervating muscle 6 without influencing transmission at the synaptic terminals of the same motor neuron that innervate the adjacent muscle 7 (Li et al., 2018a), demonstrating a remarkable degree of compartmentalized expression of PHP. We combined these manipulations to induce a simultaneous challenge of biased innervation and GluR loss using FasII overexpression combined with GluRIIA knockdown selectively on muscle 6 (referred to as M6 > FasII+GluRIIA ${ }^{\text {RNAi; }}$ see "Materials and Methods" section for details). We first tested whether the combined manipulation was successful by assaying synaptic growth and GluRIIA levels. Indeed, we observed the expected increase and decrease in bouton numbers on muscles 6 and 7 respectively, with a near absence of GluRIIA immunostaining selectively on muscle 6 (Figures 4B-F). Thus, target-specific, homeostatic challenges of biased innervation and GluR loss can be simultaneously induced by overexpressing FasII and GluRIIA ${ }^{\text {RNAi }}$ selectively on muscle 6 .

We next performed synaptic electrophysiology at both targets. On the hypo-innervated muscle 7 of M6 $>$ FasII+GluRIIA RNAi, neurotransmission was indistinguishable from M6 > FasII alone, with elevated mEPSP amplitudes, stable EPSP amplitudes, and reduced quantal content observed (Figures 4D,E). In contrast, on the hyper-innervated muscle 6 of M6 > FasII+GluRIIA ${ }^{\text {RNAi, }}$ mEPSP amplitudes were selectively reduced due to GluR knockdown, but synaptic strength was maintained at baseline levels due to a homeostatic increase in quantal content (Figures 4D,F). This demonstrates that PHP can be robustly expressed and balanced with the adaptations necessary to adjust release for hyper-innervation in a target-specific manner, without any apparent changes in transmission at adjacent synapses of the hypo-innervated muscle 7. Finally, we tested whether PHP can be acutely induced and balanced at hypo-innervated NMJs after the adjustments made at muscle 6 of M6>FasII+GluRIIA ${ }^{\text {RNAi }}$. We applied sub-blocking concentrations of the GluR venom philanthotoxin-433 (PhTx) at 
NMJs for 10 mins. This acutely induced PHP at wild type NMJs, with reduced mEPSP amplitude but EPSP amplitudes unchanged from baseline due to a rapid, homeostatic increase in quantal content (Supplementary Figures S3A-D). Application of PhTx to M6 > FasII+GluRIIA ${ }^{\text {RNAi }}$ NMJs had no significant change in mEPSP amplitude or quantal content at muscle 6 due to GluRIIA knockdown (Supplementary Figures S3A,B,D). However, PhTx application also induced robust PHP at muscle 7 NMJs in M6 > FasII+GluRIIA ${ }^{\text {RNAi }}$, with a significant reduction in mEPSP amplitude but normal EPSP amplitude due to enhanced quantal content (Supplementary Figures S3A,C,E). These results demonstrate that presynaptic release sites at terminals of the same neuron can be selectively modulated with exquisite target specificity to compensate for GluR loss and can be superimposed with the homeostatic plasticity induced by biased innervation.

\section{DISCUSSION}

Recent studies have shed light on how neurotransmission is stabilized when synaptic growth and function is challenged (Davis and Müller, 2015; Li et al., 2018b; Goel et al., 2019a,b; Frank et al., 2020). However, less is known about how this stability is maintained when neuronal terminals confront diverse and even opposing challenges in synaptic growth and function. Here, we have utilized a manipulation pioneered by Davis and Goodman (1998) to induce biased innervation and provoke target-specific plasticity and combined this with acute and chronic challenges to postsynaptic GluR function at distinct targets shared by individual neurons. These experiments have revealed two distinct target-specific mechanisms that enable stable transmission despite biased innervation, operating at either pre- or postsynaptic compartments, and that can be balanced with postsynaptic GluR perturbation. Importantly, these processes occur independently, without impacting transmission within the same neuron on neighboring synapses made on the adjacent target. This demonstrates a remarkable degree of compartmentalized autonomy in homeostatic signaling and suggests the independence of local and global homeostats that work in concert to balance synaptic strength.

\section{Target-Specific Homeostatic Scaling of Postsynaptic GluR Receptors}

We took advantage of a previously established manipulation to bias synaptic innervation using the target-specific expression of the trans-synaptic cell adhesion protein FasII (Davis and Goodman, 1998). On the hypo-innervated target, a selective upregulation in postsynaptic GluR abundance was elicited sufficient in magnitude to offset reduced neurotransmitter release and stabilize synaptic strength. This scaling of GluR abundance parallels a well-established mechanism of homeostatic synaptic plasticity in mammalian neurons termed homeostatic receptor scaling (Turrigiano, 2008; Chowdhury and Hell, 2018; Diering and Huganir, 2018). Although optogenetic activity can be used to provoke GluRs to rapidly traffic at the fly NMJ in ways that parallel the dynamics of GluRs in mammalian dendritic spines (Ljaschenko et al., 2013), the GluR scaling revealed in this study is unique. GluRs at the Drosophila NMJ are typically quite stable, and this receptor stasis may reflect a fundamental property of NMJs, where postsynaptic receptors have half-lives of $\sim 7$ days in rodents (Salpeter and Harris, 1983) and over $24 \mathrm{~h}$ in flies (Rasse et al., 2005). While NMJ receptors appear to be relatively stable under basal conditions and even in mutants in which synaptic transmission and growth are perturbed (Saitoe et al., 2001; Lee et al., 2013; Goel et al., 2019b), there is emerging evidence that specific challenges, including activity, injury, and disease, can provoke relatively rapid remodeling of neurotransmitter receptors at postsynaptic compartments of the NMJ (Rich and Lichtman, 1989; Palma et al., 2011; Ljaschenko et al., 2013; Perry et al., 2017; Goel and Dickman, 2018). The temporal regulation and dynamics of the hypo-innervation-induced GluR plasticity are unclear but likely to be intertwined with NMJ development and growth.

The induction mechanisms that enable reduced innervation to be sensed and to ultimately instruct an adaptive increase in postsynaptic GluR abundance are unclear. Two different types of motor neurons innervate most muscles in Drosophila, called type Is (phasic) and type Ib (tonic; Atwood et al., 1993; Kurdyak et al., 1994; Lnenicka and Keshishian, 2000). Differences in GluR composition have been noted at terminals of Is and Ib inputs (Schmid et al., 2008), and there is evidence that these motor neuron subtypes may possess different plasticity rules (Newman et al., 2017; Li et al., 2018b; Aponte-Santiago et al., 2020). Although no major differences in the adaptations related to hypo- and hyper-innervation were observed between Is and Ib inputs (Davis and Goodman, 1998), future work may uncover input-specific distinctions. It is notable that while hypo-innervation in the M6>FasII manipulation elicits GluR scaling, a variety of mutations that lead to synaptic undergrowth do not consistently change receptor levels (Kaufmann et al., 2002; Marqués et al., 2002; Banovic et al., 2010; Goel et al., $2019 b)$. Further, mutations that severely reduce neurotransmitter release, including synaptotagmin and complexin mutants, do not change GluR levels (Saitoe et al., 2001; Huntwork and Littleton, 2007; Lee et al., 2013). Hence, hypo-innervation and/or reduced neurotransmitter release alone is unlikely to be sufficient to induce postsynaptic GluR scaling. Rather, this form of homeostatic plasticity may be dependent on the phenomenon of biased innervation between two targets shared by a single neuron itself, implying some signaling between the motor neuron and/or the adjacent muscles is involved. What is clear is that the postsynaptic signal transduction system that mediates hypo-innervation-dependent GluR scaling is distinct from that which mediates retrograde PHP signaling, as GluR scaling can still be expressed in conditions in which postsynaptic PHP signaling is blocked (Goel and Dickman, 2018). Finally, it is interesting to note that the induction of PHP signaling is initiated by loss or blockade of GluRs, while the ultimate expression mechanism of GluR scaling involves a homeostatic upregulation in the abundance of these same GluRs at postsynaptic compartments. Thus, postsynaptic GluRs are central targets for both the induction and expression of homeostatic synaptic plasticity. 


\section{Target-Specific Modulation of Active Zones}

In contrast to the exclusively postsynaptic adaptation observed in response to reduced innervation, an entirely presynaptic mechanism stabilizes synaptic strength at hyper-innervated muscles, expressed by a target-specific reduction in the number and intensity of active zone components. Although a similar reduction in the abundance of active zone proteins at individual release sites has recently been found in mutations that cause synaptic overgrowth at the NMJ (Goel et al., 2019a,b), the adaptations observed in the case of hyper-innervation are distinct in that they are: (1) target-specific; and (2) involve a reduction in active zone density in addition to their apparent intensity. Although increased fluorescence intensity is typically interpreted to reflect enhanced protein abundance, a recent study using Localization Microscopy showed that increased active zone intensity may instead reflect a more compact nanoscopic arrangement (Mrestani et al., 2020). Nonetheless, it is remarkable that both the number and intensity of active zone components can be selectively reduced and calibrated at hyper-innervated terminals without any apparent changes at adjacent terminals shared by the same neuron on the hypo-innervated target. This suggests the intriguing possibility that target-specific modulation of active zone structure might homeostatically control a cargo delivery process at synapses. One attractive candidate pathway may involve the lysosomal adaptor Arl-8. Arl-8 regulates the delivery of synaptic vesicle and active zone cargo to synapses (Klassen et al., 2010; Vukoja et al., 2018), and was recently shown to promote the delivery of synaptic cargo necessary to remodel active zones during PHP (Goel et al., 2019a). Because active zone components are remodeled during PHP (Weyhersmüller et al., 2011; Böhme et al., 2016; Goel et al., 2017; Gratz et al., 2019) through an arl-8 dependent mechanism (Goel et al., 2019a), and PHP can be expressed at a subset of terminals with targetspecificity (Li et al., 2018a), it is tempting to speculate that Arl-8 may also be involved in the target-specific reduction in active zones following hyper-innervation.

\section{Biased Innervation, Presynaptic Homeostatic Plasticity, and Information Transfer at Synapses}

Global synaptic strength is established during development through intrinsic genetic programs and a dialogue between preand post-synaptic compartments. Robustness in this process is ensured by signaling systems that can sense and adapt to deviations outside of physiological ranges, such as reductions or enhancements in synaptic growth (Tripodi et al., 2008;

\section{REFERENCES}

Akbergenova, J., Cunningham, K. L., Zhang, Y. V., Weiss, S., and Littleton, J. T. (2018). Characterization of developmental and molecular factors underlying release heterogeneity at Drosophila synapses. Elife 7:e38268. doi: 10.7554/eLife. 38268

Allan, D. W., St Pierre, S. E., Miguel-Aliaga, I., and Thor, S. (2003). Specification of neuropeptide cell identity by the integration of retrograde BMP signaling and a combinatorial transcription
Yuan et al., 2011; Keck et al., 2013; Goel et al., 2019b,c). Superimposed on this foundation are forms of plasticity such as PHP, which appear to operate as independent homeostats to maintain stable information transfer at synapses and within neural circuits. Presynaptic terminals of a neuron, therefore, do not function as unitary computational units but are rather compartmentally specialized and flexible according to the physiologic needs of their targets during development and following homeostatic challenges. In addition to this targetspecificity, there is also evidence for input-specificity across dendrites that can homeostatically modulate strength in rodent hippocampal neurons (Katz et al., 2009; Jia et al., 2010; Stuart and Spruston, 2015; Letellier et al., 2019). This remarkable control of synaptic activity enables the flexibility to locally adjust synaptic strength through input- and target-specificity while stabilizing overall network activity and information processing.

\section{DATA AVAILABILITY STATEMENT}

The raw data supporting the conclusions of this article will be made available by the authors, without undue reservation.

\section{AUTHOR CONTRIBUTIONS}

PG and DD designed the research. PG, SN, KC, CC, and XL performed the research. PG analyzed the data. PG and DD wrote the article.

\section{FUNDING}

This work was supported by grants from the National Institutes of Health (NS111414 and NS091546) to DD. PG was supported in part by a USC Provost Graduate Research Fellowship.

\section{ACKNOWLEDGMENTS}

We acknowledge the Developmental Studies Hybridoma Bank (Iowa, USA) for antibodies used in this study, and the Bloomington Drosophila Stock Center for fly stocks (NIH P40OD018537). We thank Giwoo Kim and Sarah Perry for their technical contributions at the early stages of this project.

\section{SUPPLEMENTARY MATERIAL}

The Supplementary Material for this article can be found online at: https://www.frontiersin.org/articles/10.3389/fncel.2020.001 96/full\#supplementary-material

factor code. Cell 113, 73-86. doi: 10.1016/s0092-8674(03) 00204-6

Allan, D. W., and Thor, S. (2015). Transcriptional selectors, masters and combinatorial codes: regulatory principles of neural subtype specification. Wiley Interdiscip. Rev. Dev. Biol. 4, 505-528. doi: 10.1002/wdev.191

Aponte-Santiago, N. A., Ormerod, K. G., Akbergenova, Y., and Littleton, J. T. (2020). Synaptic plasticity induced by differential manipulation of tonic and phasic motoneurons in Drosophila. BioRxiv [Preprint]. doi: 10.1101/2020.04. 28.066696 
Atwood, H. L., Govind, C. K., and Wu, C. F. (1993). Differential ultrastructure of synaptic terminals on ventral longitudinal abdominal muscles in Drosophila larvae. J. Neurobiol. 24, 1008-1024. doi: 10.1002/neu.480240803

Atwood, H. L., and Karunanithi, S. (2002). Diversification of synaptic strength: presynaptic elements. Nat. Rev. Neurosci. 3, 497-516. doi: 10.1038/nrn876

Banovic, D., Khorramshahi, O., Owald, D., Wichmann, C., Riedt, T., Fouquet, W., et al. (2010). Drosophila neuroligin 1 promotes growth and postsynaptic differentiation at glutamatergic neuromuscular junctions. Neuron 66, 724-738. doi: 10.1016/j.neuron.2010.05.020

Béique, J. C., Na, Y., Kuhl, D., Worley, P. F., and Huganir, R. L. (2011). Arcdependent synapse-specific homeostatic plasticity. Proc. Natl. Acad. Sci. U S A 108, 816-821. doi: 10.1073/pnas.1017914108

Böhme, M. A., Beis, C., Reddy-Alla, S., Reynolds, E., Mampell, M. M., Grasskamp, A. T., et al. (2016). Active zone scaffolds differentially accumulate Unc13 isoforms to tune $\mathrm{Ca}^{2+}$ channel-vesicle coupling. Nat. Neurosci. 19, 1311-1320. doi: 10.1038/nn.4364

Böhme, M. A., McCarthy, A. W., Grasskamp, A. T., Beuschel, C. B., Goel, P., Jusyte, B., et al. (2019). Rapid active zone remodeling consolidates presynaptic potentiation. Nat. Commun. 10:1085. doi: 10.1038/s41467-019-08977-6

Branco, T., and Staras, K. (2009). The probability of neurotransmitter release: variability and feedback control at single synapses. Nat. Rev. Neurosci. 10, 373-383. doi: 10.1038/nrn2634

Brusich, D. J., Spring, A. M., James, T. D., Yeates, C. J., Helms, T. H., and Frank, C. A. (2018). Drosophila $\mathrm{Ca}_{V} 2$ channels harboring human migraine mutations cause synapse hyperexcitability that can be suppressed by inhibition of a Ca ${ }^{2+}$ store release pathway. PLoS Genet. 14:e1007577. doi: 10.1371/journal. pgen. 1007577

Calderó, J., Prevette, D., Mei, X., Oakley, R. A., Li, L., Milligan, C., et al. (1998). Peripheral target regulation of the development and survival of spinal sensory and motor neurons in the chick embryo. J. Neurosci. 18, 356-370. doi: 10.1523/JNEUROSCI.18-01-00356.1998

Chowdhury, D., and Hell, J. W. (2018). Homeostatic synaptic scaling: molecular regulators of synaptic AMPA-type glutamate receptors. F1000Res. 7:234. doi: 10.12688/f1000research.13561.1

Daniels, R. W., Collins, C. A., Gelfand, M. V., Dant, J., Brooks, E. S., Krantz, D. E., et al. (2004). Increased expression of the Drosophila vesicular glutamate transporter leads to excess glutamate release and a compensatory decrease in quantal content. J. Neurosci. 24, 10466-10474. doi: 10.1523/JNEUROSCI.300104.2004

Davis, G. W., and Goodman, C. S. (1998). Synapse-specific control of synaptic efficacy at the terminals of a single neuron. Nature 392, 82-86. doi: $10.1038 / 32176$

Davis, G. W., and Müller, M. (2015). Homeostatic control of presynaptic neurotransmitter release. Annu. Rev. Physiol. 77, 251-270. doi: 10.1146/annurev-physiol-021014-071740

Davis, G. W., Schuster, C., and Goodman, C. S. (1997). Genetic analysis of the molecular mechanisms controlling target selection: target derived Fasciclin II regulates the pattern of synapse formation. Neuron 19, 561-573. doi: 10.1016/s0896-6273(00)80372-4

DiAntonio, A. (2006). Glutamate receptors at the Drosophila neuromuscular junction. Intl. Rev. Neurobiol. 75, 165-179. doi: 10.1016/s0074-7742(06) 75008-5

Dickman, D. K., and Davis, G. W. (2009). The schizophrenia susceptibility gene dysbindin controls synaptic homeostasis. Science 326, 1127-1130. doi: 10.1126/science.1179685

Diering, G. H., and Huganir, R. L. (2018). The AMPA receptor code of synaptic plasticity. Neuron 100, 314-329. doi: 10.1016/j.neuron.2018.10.018

Fekete, A., Nakamura, Y., Yang, Y. M., Herlitze, S., Mark, M. D., DiGregorio, D. A., et al. (2019). Underpinning heterogeneity in synaptic transmission by presynaptic ensembles of distinct morphological modules. Nat. Commun. 10:826. doi: 10.1038/s41467-019-08452-2

Frank, C. A., Kennedy, M. J., Goold, C. P., Marek, K. W., and Davis, G. W. (2006). Mechanisms underlying the rapid induction and sustained expression of synaptic homeostasis. Neuron 52, 663-677. doi: 10.1016/j.neuron.2006. 09.029

Frank, C. A., James, T. D., and Müller, M. (2020). Homeostatic control of Drosophila neuromuscular junction function. Synapse 74:e22133. doi: 10.1002/syn.22133
Goel, P., Bergeron, D. D., Böhme, M., Nunnelly, L., Lehmann, M., Buser, C., et al. (2019a). Homeostatic scaling of active zone scaffolds maintains global synaptic strength. J. Cell Biol. 218, 1706-1724. doi: 10.1083/jcb.201807165

Goel, P., Khan, M., Howard, S., Kim, G., Kiragasi, B., Kikuma, K., et al. (2019b). A screen for synaptic growth mutants reveals mechanisms that stabilize synaptic strength. J. Neurosci. 39, 4051-4065. doi: 10.1523/JNEUROSCI.2601-18.2019

Goel, P., Li, X., and Dickman, D. (2019c). Estimation of the readily releasable synaptic vesicle pool at the Drosophila larval neuromuscular junction. Bio Protoc. 9:e3127. doi: 10.21769/bioprotoc.3127

Goel, P., and Dickman, D. (2018). Distinct homeostatic modulations stabilize reduced postsynaptic receptivity in response to presynaptic DLK signaling. Nat. Commun. 9:1856. doi: 10.1038/s41467-018-04270-0

Goel, P., Li, X., and Dickman, D. (2017). Disparate postsynaptic induction mechanisms ultimately converge to drive the retrograde enhancement of presynaptic efficacy. Cell Rep. 21, 2339-2347. doi: 10.1016/j.celrep.2017.10.116

Graf, E. R., Daniels, R. W., Burgess, R. W., Schwarz, T. L., and DiAntonio, A. (2009). Rab3 dynamically controls protein composition at active zones. Neuron 64, 663-677. doi: 10.1016/j.neuron.2009.11.002

Gratz, S. J., Goel, P., Bruckner, J. J., Hernandez, R. X., Khateeb, K., Macleod, G., et al. (2019). Endogenous tagging reveals differential regulation of $\mathrm{Ca}^{2+}$ channels at single AZs during presynaptic homeostatic potentiation and depression. J. Neurosci. 39, 2416-2429. doi: 10.1523/JNEUROSCI.3068-18.2019

Grillo, F. W., Neves, G., Walker, A., Vizcay-Barrena, G., Fleck, R. A., Branco, T., et al. (2018). A distance-dependent distribution of presynaptic boutons tunes frequency-dependent dendritic integration. Neuron 99, 275-282. doi: 10.1016/j. neuron.2018.06.015

Guerrero, G., Reiff, D. F., Agarwal, G., Ball, R. W., Borst, A., Goodman, C. S., et al. (2005). Heterogeneity in synaptic transmission along a Drosophila larval motor axon. Nat. Neurosci. 8, 1188-1196. doi: 10.1038/nn1526

Henry, F. E., McCartney, A. J., Neely, R., Perez, A. S., Carruthers, C. J., Stuenkel, E. L., et al. (2012). Retrograde changes in presynaptic function driven by dendritic mTORC1. J. Neurosci. 32, 17128-17142. doi: 10.1523/JNEUROSCI.2149-12.2012

Hou, Q., Zhang, D., Jarzylo, L., Huganir, R. L., and Man, H. Y. (2008). Homeostatic regulation of AMPA receptor expression at single hippocampal synapses. Proc. Natl. Acad. Sci. U S A 105, 775-780. doi: 10.1073/pnas.0706447105

Huntwork, S., and Littleton, J. T. (2007). A complexin fusion clamp regulates spontaneous neurotransmitter release and synaptic growth. Nat. Neurosci. 10, 1235-1237. doi: 10.1038/nn1980

Jakawich, S. K., Nasser, H. B., Strong, M. J., McCartney, A. J., Perez, A. S., Rakesh, N., et al. (2010). Local presynaptic activity gates homeostatic changes in presynaptic function driven by dendritic BDNF synthesis. Neuron 68, 1143-1158. doi: 10.1016/j.neuron.2010.11.034

Jia, H., Rochefort, N. L., Chen, X., and Konnerth, A. (2010). Dendritic organization of sensory input to cortical neurons in vivo. Nature 464, 1307-1312. doi: 10.1038/nature08947

Katz, P. S., Kirk, M. D., and Givind, C. K. (1993). Facilitation and depression at different branches of the same motor axon: evidence for presynaptic differences in release. J. Neurosci. 13, 3075-3089. doi: 10.1523/JNEUROSCI.13-07-03 075.1993

Katz, Y., Menon, V., Nicholson, D. A., Geinisman, Y., Kath, W. L., and Spruston, N. (2009). Synapse distribution suggests a two-stage model of dendritic integration in CA1 pyramidal neurons. Neuron 63, 171-177. doi: 10.1016/j.neuron.2009.06.023

Kaufmann, N., DeProto, J., Ranjan, R., Wan, H., and Van Vactor, D. (2002). Drosophila liprin- $\alpha$ and the receptor phosphatase Dlar control synapse morphogenesis. Neuron 34, 27-38. doi: 10.1016/s0896-6273(02)00643-8

Keck, T., Keller, G. B., Jacobsen, R. I., Eysel, U. T., Bonhoeffer, T., and Hubener, M. (2013). Synaptic scaling and homeostatic plasticity in the mouse visual cortex in vivo. Neuron 80, 327-334. doi: 10.1016/j.neuron.2013.08.018

Kikuma, K., Li, X., Kim, D., Sutter, D., and Dickman, D. K. (2017). Extended synaptotagmin localizes to presynaptic ER and promotes neurotransmission and synaptic growth in Drosophila. Genetics 207, 993-1007. doi: 10.1534/genetics.117.300261

Kiragasi, B., Wondolowski, J., Li, Y., and Dickman, D. K. (2017). A presynaptic glutamate receptor subunit confers robustness to neurotransmission and homeostatic potentiation. Cell Rep. 19, 2694-2706. doi: 10.1016/j.celrep.2017. 06.003 
Klassen, M. P., Wu, Y. E., Maeder, C. I., Nakae, I., Cueva, J. G., Lehrman, E. K., et al. (2010). An Arf-like small G protein, ARL-8, promotes the axonal transport of presynaptic cargoes by suppressing vesicle aggregation. Neuron 66, 710-723. doi: 10.1016/j.neuron.2010.04.033

Kurdyak, P., Atwood, H. L., Stewart, B. A., and Wu, C. F. (1994). Differential physiology and morphology of motor axons to ventral longitudinal muscles in larval Drosophila. J. Comp. Neurol. 350, 463-472. doi: 10.1002/cne.903500310

Lee, J., Guan, Z., Akbergenova, Y., and Littleton, J. T. (2013). Genetic analysis of synaptotagmin C2 domain specificity in regulating spontaneous and evoked neurotransmitter release. J. Neurosci. 33, 187-200. doi: 10.1523/JNEUROSCI. 3214-12.2013

Letellier, M., Levet, F., Thoumine, O., and Goda, Y. (2019). Differential role of pre- and postsynaptic neurons in the activity-dependent control of synaptic strengths across dendrites. PLoS Biol. 17:e2006223. doi: 10.1371/journal.pbio. 2006223

Li, X., Goel, P., Chen, C., Angajala, V., Chen, X., and Dickman, D. (2018a). Synapse-specific and compartmentalized expression of presynaptic homeostatic potentiation. Elife 7:e34338. doi: 10.7554/eLife.34338

Li, X., Goel, P., Wondolowski, J., Paluch, J., and Dickman, D. (2018b). A glutamate homeostat controls the presynaptic inhibition of neurotransmitter release. Cell Rep. 23, 1716-1727. doi: 10.1016/j.celrep.2018.03.130

Ljaschenko, D., Ehmann, N., and Kittel, R. J. (2013). Hebbian plasticity guides maturation of glutamate receptor fields in vivo. Cell Rep. 3, 1407-1413. doi: 10.1016/j.celrep.2013.04.003

Lnenicka, G. A., and Keshishian, H. (2000). Identified motor terminals in Drosophila larvae show distinct differences in morphology and physiology. J. Neurobiol. 43, 186-197. doi: 10.1002/(sici)1097-4695(200005)43:2<186::aidneu8 $>3.0 . c 0 ; 2-n$

Marqués, G., Bao, H., Haerry, T. E., Shimell, M. J., Duchek, P., Zhang, B., et al. (2002). The Drosophila BMP type II receptor wishful thinking regulates neuromuscular synapse morphology and function. Neuron 33, 529-543. doi: 10.1016/s0896-6273(02)00595-0

Melom, J. E., Akbergenova, Y., Gavornik, J. P., and Littleton, J. T. (2013). Spontaneous and evoked release are independently regulated at individual active zones. J. Neurosci. 33, 17253-17263. doi: 10.1523/JNEUROSCI.3334-13. 2013

Mrestani, A., Kollmannsberger, P., Pauli, M., Repp, F., Kittel, R. J., Eilers, J., et al. (2020). Active zone compaction in presynaptic homeostatic potentiation. BioRxiv [Preprint]. doi: 10.1101/802843

Newman, Z. L., Hoagland, A., Aghi, K., Worden, K., Levy, S. L., Son, J. H., et al. (2017). Input-specific plasticity and homeostasis at the Drosophila larval neuromuscular junction. Neuron 93, 1388-1404. doi: 10.1016/j.neuron.2017. 02.028

O’Rourke, N. A., Weiler, N. C., Micheva, K. D., and Smith, S. J. (2012). Deep molecular diversity of mammalian synapses: why it matters and how to measure it. Nat. Rev. Neurosci. 13, 365-379. doi: 10.1038/nrn3170

Palma, E., Inghilleri, M., Conti, L., Deflorio, C., Frasca, V., Manteca, A., et al. (2011). Physiological characterization of human muscle acetylcholine receptors from ALS patients. Proc. Natl. Acad. Sci. U S A 108, 20184-20188. doi: 10.1073/pnas.1117975108

Park, H., and Poo, M. M. (2013). Neurotrophin regulation of neural circuit development and function. Nat. Rev. Neurosci. 14, 7-23. doi: 10.1038/nrn3379

Perry, S., Han, Y., Das, A., and Dickman, D. K. (2017). Homeostatic plasticity can be induced and expressed to restore synaptic strength at neuromuscular junctions undergoing ALS-related degeneration. Hum. Mol. Genet. 26, 4153-4167. doi: 10.1093/hmg/ddx304

Qin, G., Schwarz, T., Kittel, R. J., Schmid, A., Rasse, T. M., Kappei, D., et al. (2005). Four different subunits are essential for expressing the synaptic glutamate receptor at neuromuscular junctions of Drosophila. J. Neurosci. 25, 3209-3218. doi: 10.1523/JNEUROSCI.4194-04.2005

Rasse, T. M., Fouquet, W., Schmid, A., Kittel, R. J., Mertel, S., Sigrist, C. B., et al. (2005). Glutamate receptor dynamics organizing synapse formation in vivo. Nat. Neurosci. 8, 898-905. doi: 10.1038/nn1484

Regehr, W. G. (2012). Short-term presynaptic plasticity. Cold Spring Harb. Perspect. Biol. 4:a005702. doi: 10.1101/cshperspect.a005702

Rich, M. M., and Lichtman, J. W. (1989). In vivo visualization of pre- and postsynaptic changes during synapse elimination in reinnervated mouse muscle. J. Neurosci. 5, 1781-1805. doi: 10.1523/JNEUROSCI.09-05-01781.1989
Rudolph, S., Tsai, M. C., von Gersdorff, H., and Wadiche, J. I. (2015). The ubiquitous nature of multivesicular release. Trends Neurosci. 38, 428-438. doi: 10.1016/j.tins.2015.05.008

Saitoe, M., Schwarz, T. L., Umbach, J. A., Gundersen, C. B., and Kidokoro, Y. (2001). Absence of junctional glutamate receptor clusters in Drosophila mutants lacking spontaneous transmitter release. Science 293, 514-517. doi: $10.1126 /$ science. 1061270

Salpeter, M. M., and Harris, R. (1983). Distribution and turnover rate of acetylcholine receptors throughout the junction folds at a vertebrate neuromuscular junction. J. Cell Biol. 96, 1781-1785. doi: 10.1083/jcb. 96.6.1781

Schmid, A., Hallermann, S., Kittel, R. J., Khorramshahi, O., Frölich, A. M., Quentin, C., et al. (2008). Activity-dependent site-specific changes of glutamate receptor composition in vivo. Nat. Neurosci. 11, 659-666. doi: 10.1038/nn.2122

Schotzinger, R. J., and Landis, S. C. (1990). Acquisition of cholinergic and peptidergic properties by sympathetic innervation of rat sweat glands requires interaction with normal target. Neuron 5, 91-100. doi: 10.1016/08966273(90)90037-g

Stewart, B. A., Atwood, H. L., Renmger, J. J., Wang, J., and Wu, C. F. (1994). Improved stability of Drosophila larval neuromuscular prepa- rations in haemolymph-like physiological solutions. J. Comp. Physiol. 175, 179-191. doi: $10.1007 / \mathrm{bf} 00215114$

Stuart, G. J., and Spruston, N. (2015). Dendritic integration: 60 years of progress. Nat. Neurosci. 18, 1713-1721. doi: 10.1038/nn.4157

Sutton, M. A., Ito, H. T., Cressy, P., Kempf, C., Woo, J. C., and Schuman, E. M. (2006). Miniature neurotransmission stabilizes synaptic function via tonic suppression of local dendritic protein synthesis. Cell 125, 785-799. doi: 10.1016/j.cell.2006.03.040

Tripodi, M., Evers, J. F., Mauss, A., Bate, M., and Landgraf, M. (2008). Structural homeostasis: compensatory adjustments of dendritic arbor geometry in response to variations of synaptic input. PLoS Biol. 6:e260. doi: 10.1371/journal. pbio. 0060260

Turrigiano, G. (2012). Homeostatic synaptic plasticity: local and global mechanisms for stabilizing neuronal function. Cold Spring Harb. Perspect. Biol. 4:a005736. doi: 10.1101/cshperspect.a005736

Turrigiano, G. G. (2008). The self-tuning neuron: synaptic scaling of excitatory synapses. Cell 135, 422-435. doi: 10.1016/j.cell.2008.10.008

Turrigiano, G. G., and Nelson, S. B. (2004). Homeostatic plasticity in the developing nervous system. Nat. Rev. Neurosci. 5, 97-107. doi: $10.1038 / \mathrm{nrn} 1327$

Vitureira, N., and Goda, Y. (2013). The interplay between Hebbian and homeostatic synaptic plasticity. J. Cell Biol. 203, 175-186. doi: 10.1083/jcb. 201306030

Vitureira, N., Letellier, M., White, I. J., and Goda, Y. (2011). Differential control of presynaptic efficacy by postsynaptic N-cadherin and $\beta$-catenin. Nat. Neurosci. 15, 81-89. doi: 10.1038/nn.2995

Vukoja, A., Rey, U., Petzoldt, A. G., Ott, C., Vollweiter, D., Quentin, C., et al. (2018). Presynaptic biogenesis requires axonal transport of lysosomerelated vesicles. Neuron 99, 1216.e7-1232.e7. doi: 10.1016/j.neuron.2018. 08.004

Weyhersmüller, A., Hallermann, S., Wagner, N., and Eilers, J. (2011). Rapid active zone remodeling during synaptic plasticity. J. Neurosci. 31, 6041-6052. doi: 10.1523/JNEUROSCI.6698-10.2011

Yuan, Q., Xiang, Y., Yan, Z., Han, C., Jan, L. Y., and Jan, Y. N. (2011). Lightinduced structural and functional plasticity in Drosophila larval visual system. Science 333, 1458-1462. doi: 10.1126/science.1207121

Conflict of Interest: The authors declare that the research was conducted in the absence of any commercial or financial relationships that could be construed as a potential conflict of interest.

Copyright $\odot 2020$ Goel, Nishimura, Chetlapalli, Li, Chen and Dickman. This is an open-access article distributed under the terms of the Creative Commons Attribution License (CC BY). The use, distribution or reproduction in other forums is permitted, provided the original author(s) and the copyright owner(s) are credited and that the original publication in this journal is cited, in accordance with accepted academic practice. No use, distribution or reproduction is permitted which does not comply with these terms. 